\title{
FIRST DEMONSTRATION OF STAGED LASER ACCELERATION
}

\author{
W. D. Kimura, ${ }^{\dagger}$ L. P. Campbell, C. E. Dilley, S. C. Gottschalk, D. C. Quimby, \\ STI Optronics, Inc., Bellevue, WA 98004, USA \\ M. Babzien, I. Ben-Zvi, J. C. Gallardo, K. P. Kusche, I. V. Pogorelsky, J. Skaritka, \\ A. van Steenbergen, V. Yakimenko, BNL, Upton, NY 11973, USA \\ D. B. Cline, P. He, Y. Liu, UCLA, CA, 90024, USA \\ L. C. Steinhauer, Redmond Plasma Physics Laboratory, Redmond, WA 98052, USA \\ R. H. Pantell, Stanford University, CA 94305, USA
}

\begin{abstract}
Two independently-driven laser accelerators were operated together in series for the first time in a proof-ofprinciple experiment to demonstrate staging. The ability to stage together these devices is important for eventually building practical laser-driven accelerators. The laser accelerators consisted of two identical inverse free electron lasers (IFEL), where the first IFEL served as a prebuncher, which created $\sim 3$-fs long microbunches that were accelerated by the second IFEL. Precise and stable control of the phasing between the microbunches and laser wave inside the second IFEL was demonstrated. The effects of overmodulation of the prebuncher were also investigated. In all cases there was good agreement with the model. Additional details of the microbunch characteristics could be inferred by using the model. Plans for demonstrating monoenergetic laser acceleration are also presented.
\end{abstract}

\section{DESCRIPTION OF EXPERIMENT}

Routine laser acceleration of electrons is being performed around the world with gradients $>100 \mathrm{MeV} / \mathrm{m}$ demonstrated [1]. However, these high gradients have been limited to millimeter distances and generally involve a single interaction with the laser beam. To achieve high net energy gain requires staging the process whereby the electrons are repeatedly accelerated by the laser field in a manner similar to microwave accelerators.

The goal of the Staged Electron Laser Acceleration (STELLA) experiment was to demonstrate staging between two laser accelerators [2]. A pair of inverse free electron lasers (IFEL) [3] are used for the laser accelerators. The first IFEL (IFEL1) acted as an energy modulator of the $e$-beam (i.e., prebuncher), which resulted in the creation of femtosecond microbunches. The second IFEL (IFEL2) then accelerated these microbunches. A key issue is achieving proper phase synchronization between the microbunches formed by the prebuncher and the laser field in the accelerator (IFEL2).

In an IFEL, the $e$-beam copropagates with a laser beam inside a magnetic array called a wiggler or undulator. The undulator causes the electron trajectory to oscillate in the

\footnotetext{
†wkimura@stioptronics.com
}

plane of the laser beam electric field as illustrated in Fig. 1 , thereby projecting a component of this field in the direction of the electron motion. Depending on the sign of the electric field (i.e., its phase relative to the electrons), the field can accelerate or decelerate the electrons.

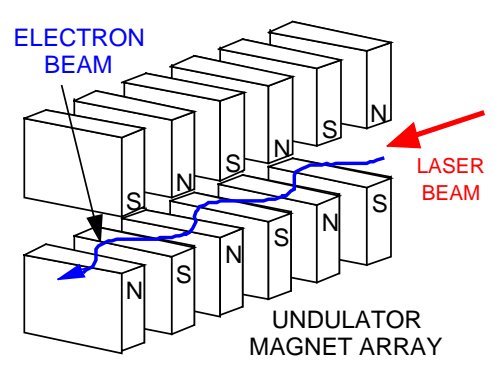

Figure 1: Illustration for an IFEL based upon a planar undulator.

The oscillatory trajectory of the electrons within the undulator eventually limits the amount of acceleration because of synchrotron radiation losses. Indeed, much higher acceleration gradients are possible using other laser acceleration mechanisms, such as laser wakefield acceleration (LWFA) [1]. Nevertheless, IFELs provided a convenient means for this proof-of-principle demonstration, and the principles and techniques developed during this experiment can be applied to other laser acceleration processes.

A schematic of the STELLA experiment is depicted in Fig. 2. The ATF $\mathrm{CO}_{2}$ laser beam is split into two beams with approximately $24 \mathrm{MW}$ sent to the prebuncher (IFEL1) and up to $300 \mathrm{MW}$ sent to the accelerator (IFEL2). Axicon lenses convert the Gaussian-profile laser beam into an annular one. Focusing telescopes focus the beams at the center of each undulator. An adjustable optical delay stage permits changing the phase of the laser beam entering IFEL2 relative to the beam entering IFEL1. Each laser beam enters the beam line vacuum pipe through windows and is directed to the undulators using in-vacuum mirrors with central holes for transmission of the $e$-beam. The separation distance between the exit of IFEL1 and the entrance to IFEL2 is 2 $\mathrm{m}$. At the end of the beam line is a spectrometer featuring 
a wide energy acceptance $( \pm 20 \%)$ capable of measuring the entire electron spectrum in a single shot.

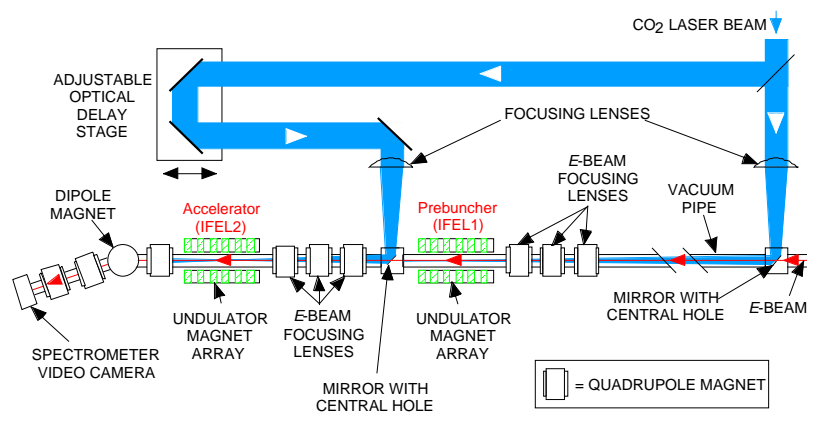

Figure 2: Schematic of STELLA experiment.

\section{EXPERIMENTAL RESULTS AND COMPARISON WITH MODEL}

\subsection{Description of Model}

The model is based upon a classical FEL simulation [4] and includes 3D effects, such as emittance and beam misalignments, and 1D space-charge effects. It ray-traces the electron trajectories through the drift region between IFEL1 and IFEL2. The parameters for the STELLA experiment that were used in the model are listed in Table I.

Table 1: Parameters for STELLA experiment.

\begin{tabular}{|l|l|}
\hline \multicolumn{1}{|c|}{ Parameter } & \multicolumn{1}{c|}{ Value } \\
\hline$E$-beam energy & $45.6 \mathrm{MeV}^{*}$ \\
\hline$E$-beam energy spread & $0.04 \%(1 \sigma)$ \\
\hline$E$-beam macropulse length & $\approx 3 \mathrm{ps}$ \\
\hline Wiggler wavelength & $3.3 \mathrm{~cm}$ \\
\hline Wiggler $K$ parameter & 2.9 \\
\hline Laser wavelength & $10.6 \mu \mathrm{m}$ \\
\hline Laser pulse length & $\approx 180 \mathrm{ps}$ \\
\hline Laser peak power to prebuncher & $24 \mathrm{MW}$ \\
\hline Laser peak power to accelerator & $200-300 \mathrm{MW}$ \\
\hline
\end{tabular}

*Energy required to be resonant with undulator.

\subsection{Prebunching Predictions}

The $e$-beam pulse length is much shorter than the laser pulse length. This means the electrons enter the prebuncher distributed uniformly over all phases of the laser field inside the undulator. This is depicted in Fig 3(a), which is a model simulation for the prebuncher. Hence, the laser imparts a sinusoidal energy modulation on the $e$-beam [see Fig. 3(b)] with an amplitude of $\approx \pm 0.5 \%$ for $24 \mathrm{MW}$ into IFEL1. This amount of modulation is chosen so that after drifting $2 \mathrm{~m}$ to IFEL2, the fast electrons catch up with the slow ones resulting in longitudinal density bunching of the electrons into microbunches [see Fig. 3(c)]. Because this modulation is induced by the laser field, these microbunches have bunch lengths a fraction of the laser wavelength. And, since the laser wavelength $(10.6 \mu \mathrm{m})$ is much shorter than the $e$ - beam pulse length, a train of $\sim 3$-fs microbunches is formed with each microbunch spaced apart by the laser wavelength $(\sim 30 \mathrm{fs})$.

\subsection{Staging Experimental Results}

Figure 4 shows false-color raw video images from the spectrometer camera. Figure 4(a) shows the energy spectrum for the $e$-beam only. After modulation by the prebuncher, the spectrum changes into a symmetric double-peaked one shown in Fig. 4(b). With the prebuncher and accelerator both operating and the phase delay between the laser beams entering the prebuncher and accelerator adjusted for maximum acceleration, a clear peak can be seen in the spectrum [see Fig. 4(c)] representing the accelerated microbunches. By changing the phase delay by $180^{\circ}$, these microbunches can be decelerated [see Fig. 4(d)].

The microbunches can be characterized by examining how the $e$-beam energy spectrum is altered when the microbunches interact with the laser beam inside the accelerator (IFEL2). The model predicts distinctive changes in the energy spectrum depending on the microbunch characteristics and the phase at which they enter the accelerator.
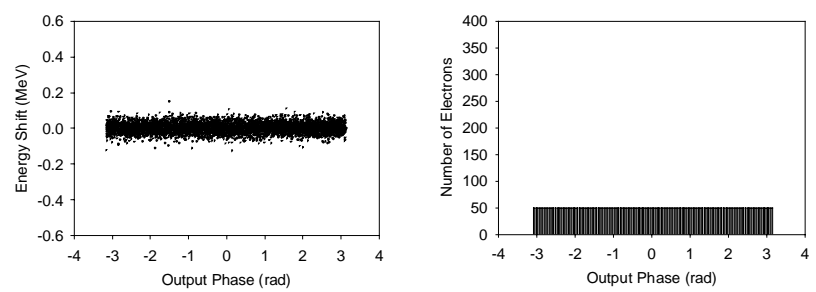

(a) Before prebuncher
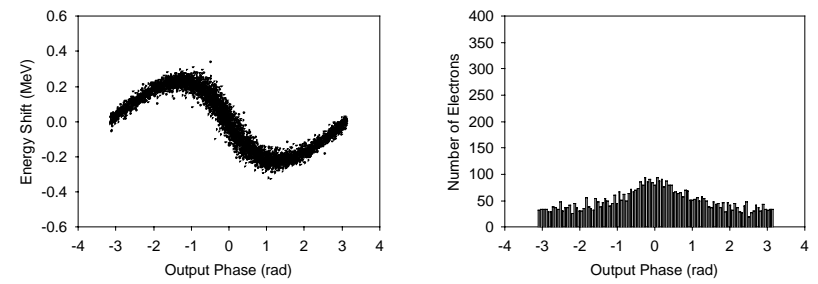

(b) At prebuncher exit
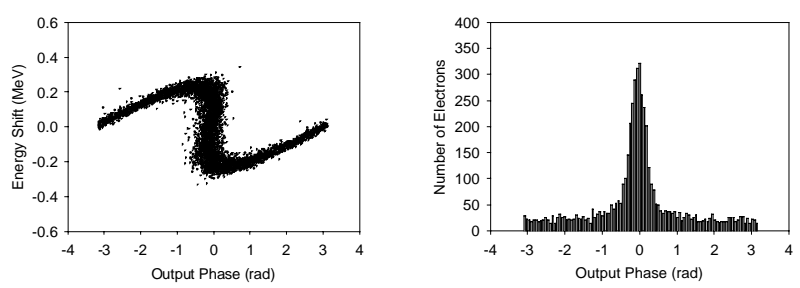

(c) After drifting to optimum bunching point

Figure 3: Model simulations for the prebuncher showing the electron energy-phase distribution and longitudinal density distribution. (a) At entrance to prebuncher. (b) At exit to prebuncher. (c) After drifting $2 \mathrm{~m}$ to the accelerator.

Figure 5 compares the model predictions with the experimental data for the phase delay set at near- 
maximum acceleration and 200 MW delivered to the accelerator. The raw video signal of the energy spectrum is shown in Fig. 5(d); its line profile is plotted in Fig. 5(c). We see there is good agreement between the model spectrum and the line profile. (The model and data energy spectra are adjusted to have equal areas.) Figure 5(a) shows the electron phase distribution that gave rise to the model energy spectrum in Fig. 5(c). A concentration of electrons representing the microbunch can be clearly seen. These electrons projected onto the phase axis [see Fig. 5(b)] indicate that the microbunch length is $\sim 0.8 \mu \mathrm{m}$ long (FWHM) corresponding to $\sim 2.7 \mathrm{fs}$ in duration.

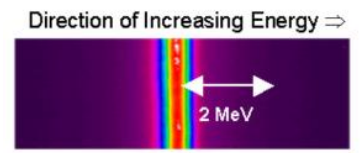

(a) E-beam only

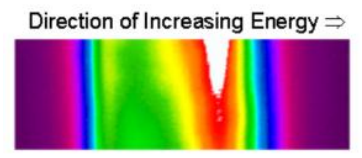

(c) Prebuncher +

Accelerator at phase for maximum acceleration

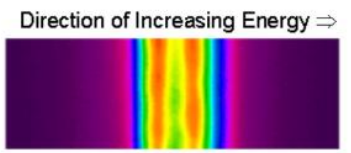

(b) Prebuncher only

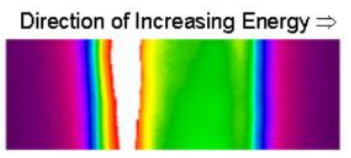

(d) Prebuncher + Accelerator at phase for maximum deceleration
Figure 4: Spectrometer output showing false-color images of the $e$-beam energy spectrum with white representing saturation. (a) With $e$-beam only. (b) With prebuncher only. (c) With prebuncher and accelerator, and phase delay set for near-maximum acceleration. (d) With prebuncher and acceleration, and phase delay set $180^{\circ}$ from (c).

Changing the phase delay between the laser beams driving the IFELs causes the microbunch to move within the energy spectrum as demonstrated in Fig. 6. We see once again that the model and data agree well at all phase positions.

Acceleration of well-formed microbunches can be disrupted if the conditions are not correct. For example, sending too little or too much laser power to the prebuncher can cause the microbunches to have their maximum density distribution (i.e., smallest bunch length) either downstream or upstream of the accelerator, respectively. Figure 7 shows the data and model predictions for the case when too much laser power is delivered, i.e., there is overmodulation occurring in the prebuncher.

The laser powers driving the two IFELs in Fig. 7 are comparable to each other. This results in a breaking apart of the concentration of electrons seen previously in Fig. 5(a) into separate bands of electrons [see Fig. 7(a)]. The effect of these bands is the appearance of four peaks within the energy spectrum as confirmed by the data [see Fig. 7(c)]. The net result is loss of the microbunch [see Fig. 7(b)].

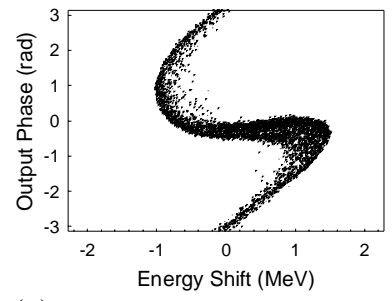

(a)

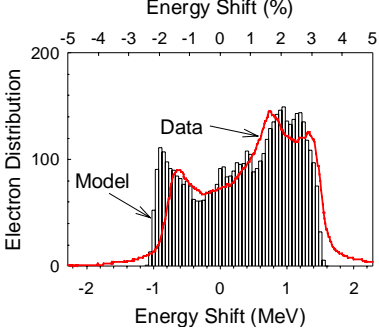

(c)

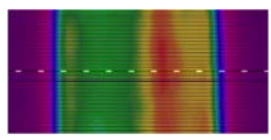

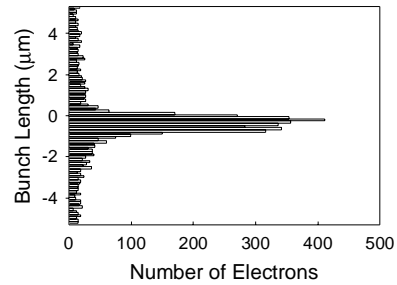

(b)
Figure 5: Comparison of data with model for the case of near-maximum acceleration of the microbunch. (a) Energy-phase. (b) Longitudinal density distribution. (c) Energy spectrum. (d) Raw video image from spectrometer.
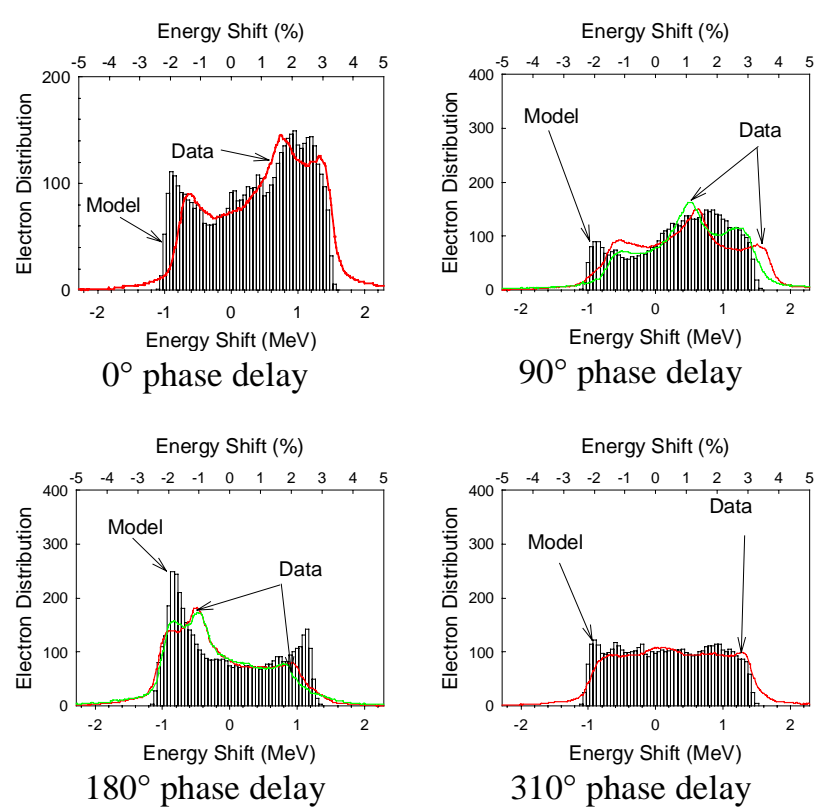

Figure 6: Model and data energy spectra as a function phase delay between the laser beams entering the two IFELs. Zero phase has been arbitrarily chosen to correspond to maximum acceleration.

\subsection{STELLA-II Program}

The staging process demonstrated during STELLA was remarkably stable. Even though there was no active phase stabilization used and the various mirrors directing the laser beams were separated by many meters, phase 
synchronization could be maintained over periods of many minutes. In order to help improve this phase stability, the current program, called STELLA-II, will modify the experiment so that a single laser beam drives both the prebuncher and accelerator. This entirely eliminates any phase jitter related to using separate laser beams to drive the IFELs. This new system is depicted in Fig. 8.

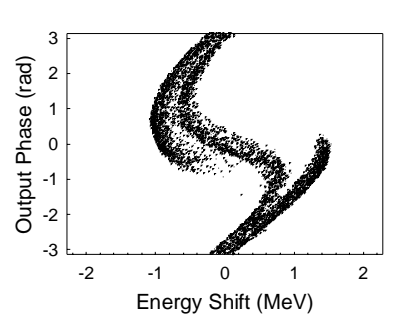

(a)

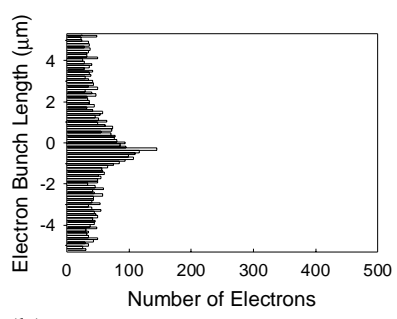

(b)

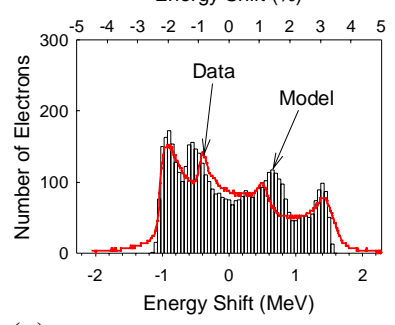

(c)

(d)

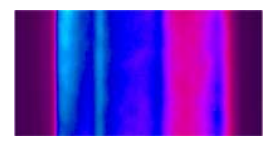

\section{Spectrometer}

output

Figure 7: Comparison of model and data for the case of 90 MW sent to the prebuncher and $115 \mathrm{MW}$ to the accelerator. (a) Energy-phase. (b) Longitudinal density distribution. (c) Energy spectrum. (d) Raw video image from spectrometer.

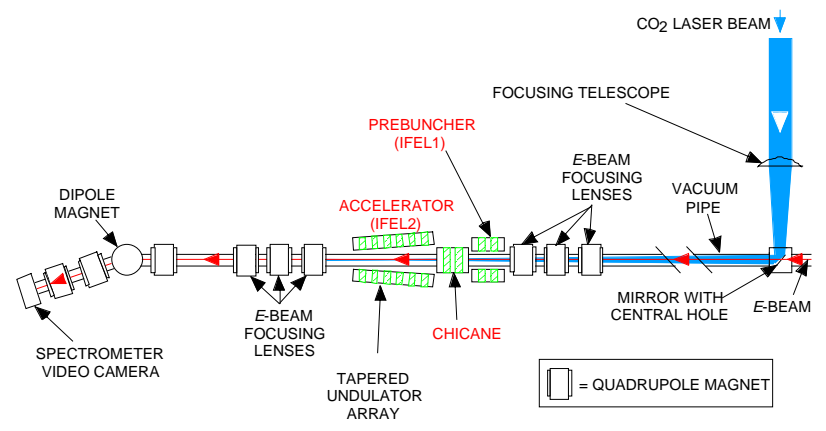

Figure 8: Schematic of planned STELLA-II experiment.

STELLA-II will be using the upgraded ATF laser capable of delivering several hundred gigawatts of peak power. Because this laser power is much larger than needed to drive the prebuncher, the undulator for the prebuncher will be replaced with a 3-period electromagnet that is intentionally detuned in order to still provide a modulation of $\pm 0.5 \%$ even with 100 's of GW of laser power passing through it. Using a single laser also

requires a minimum drift space between the IFELs. Thus, a short-length magnetic chicane will be utilized between the new prebuncher and accelerator. The laser beam will also be passing through the chicane. Therefore, to minimize the possibility of additional laser-induced modulation occurring, the chicane will be oriented with its magnetic field orthogonal to the prebuncher.

A final important modification during STELLA-II will be to use a tapered undulator for the accelerator. This will permit better trapping of the microbunches and greater energy gain using the higher laser power that will be available. A model simulation for STELLA-II is shown in Fig. 9.

With $100 \mathrm{GW}$ of laser power and $25 \%$ energy taper in the accelerator undulator, the model predicts an energy gain of $\approx 13 \mathrm{MeV}$ for a $45.6 \mathrm{MeV} e$-beam [see Fig. 9(c)]. As can be seen in Fig. 9(a), the microbunch has been cleanly separated from the unaccelerated electrons and rotates in phase-space approximately $3 / 4$ of a synchrotron period. In addition, the accelerated microbunch has an energy spread of $1.2 \%$ FWHM. Hence, STELLA-II will demonstrate monoenergetic acceleration of the microbunches. Like staging, monoenergetic acceleration is an important requirement for practical accelerators.

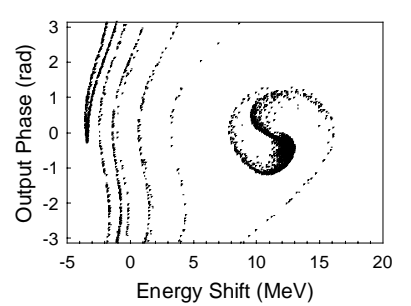

(a)

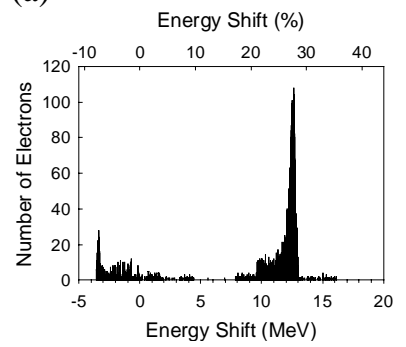

(c)

Figure 9: Model predictions for STELLA-II for $100 \mathrm{GW}$ laser power and $25 \%$ taper in the accelerator undulator.

\section{CONCLUSIONS}

A number of noteworthy accomplishments occurred during the STELLA program: 1) first demonstration of a laser-driven prebuncher staged together with a laserdriven accelerator; 2) first direct measurement of $\sim 3$-fs microbunches produced by a laser external to a wiggler; 3) first demonstration of acceleration of laser-generated microbunches with stable phase control maintained over periods of many minutes; and 4) first demonstration of laser-accelerated microbunches where a large portion of the electrons receive maximum energy gain. This last accomplishment is particularly noteworthy since the 
accelerated electrons in laser acceleration experiments to date typically exhibit wide energy distributions with only a relatively small number of electrons experiencing a narrow energy gain.

The close agreement between the data and model implies that the experimental results are a product of a systematic approach and thorough understanding of the process; the microbunch behavior is understood at every stage and phase; and the model can be confidently used for further predictions and optimization.

The experiment also showed the benefits of using a long wavelength laser which eases stability and rephasing requirements, and lessens sensitivity to bunch smearing effects.

STELLA-II promises to see further improvements in the quality of the microbunch trapping and the demonstration of monoenergetic acceleration. This will help bring this technology closer to someday realizing practical laser accelerator systems.

\section{ACKNOWLEDGEMENTS}

The authors wish to acknowledge Dr. J. R. Fontana for his technical advice during the initial planning of this experiment, and Dr. Xijie Wang and the staff at the ATF for their support. This work was supported by the U.S. Department of Energy, Grant Nos. DE-FG03-98ER41061, DE-AC02-98CH10886, and DE-FG03-92ER40695.

\section{REFERENCES}

[1] W. P. Leemans and E. Esarey, in Proceedings of Advanced Accelerator Concepts 8th Workshop, Baltimore, MD, Jul. 5-11, 1998, edited by W. Lawson, C. Bellamy and D. Brosius (American Institute of Physics, New York, 1999), p. 174.

[2] W. D. Kimura, et al., Phys. Rev. Lett. 86, p. 4041 (2001).

[3] R. B. Palmer, J. Appl. Phys. 43, p. 3014 (1972).

[4] D. C. Quimby, J. M. Slater and J. P. Wilcoxon, IEEE J. Quan. Elect. QE-21, p. 979 (1985). 